\title{
Vortex Dynamics, Combinatorics and Statistics
}

\author{
Chjan C. Lim \\ Dept. of Mathematical Sciences \\ RPI, Troy, NY 12180, USA
}

\begin{abstract}
This report represents an overview of the interconnections between the dynamics of large vortex systems, combinatorics, n-body problems and statistical mechanics. The combinatorial perturbation method for the $2 \mathrm{D}$ vortex problem is discussed; the essential combinatorial symplectic transformations to Jacobi-type variables which are based on a binary tree algorithm, is introduced and extended to the 3D vortex problem. Combinatorial and graph-theoretic results which are motivated by the computational needs of the vortex problem, are mentioned. They include new results on sign-nonsingular patterns and noneven digraphs. A simplified singular limit of the 3D Hamiltonian for vortex dynamics is derived and its basic properties discussed. The 2- and 3-body problems in this simple model is studied.
\end{abstract}

\section{Introduction}

In this report, I will discuss some aspects of $2 \mathrm{D}$ and 3D vortex dynamics which are pertinent to the numerical computation of inviscid vortical flows, and the statistical study of turbulence. The following Hamiltonian formulation for 3D Euler flows is the basis for a number of questions and results which can further the above aims. In the computational problem, assuming a particle method is used, one needs to track a large number $N \sim O\left(10^{4}\right)$ particles. A Hamiltonian formulation is optimal in many ways, not the least of which is the relatively easy checking of conservation laws. Also, nice canonical transformations that massage the high-degree of freedom ODE's, are available to ease the numerical computation of clustered vortical flows, for example. An extra bonus of a Hamiltonian formulation is the set of standard ideas and tools from statistical mechanics for the statistical study of turbulent flows (cf. Chorin [1]).

\section{Hamiltonian formulation}

Consider the recent Hamiltonian formulation of 3D ideal fluids given in [1]. Starting with the definition of magnetisation $\mathbf{M}$ in terms of the velocity $u$

$$
\begin{aligned}
\mathbf{M} & =\mathbf{u}+\nabla \phi, \quad \mathbf{M}=\left(M_{1}, M_{2}, M_{3}\right) \\
\triangle \phi & =\nabla \cdot \mathbf{M}, \quad \mathbf{u}=\left(u_{1}, u_{2}, u_{3}\right)
\end{aligned}
$$

one derives the equations of motion

$$
\frac{D M_{i}}{D t}=-M_{j} \partial_{i} u_{j}
$$

which implies Euler's equation where the pressure is identified with

$$
\frac{D \phi}{D t}+u^{2} / 2
$$


Under the usual discrete approximation for vortex methods (cf. Chorin [1]), the approximate magnetisation is given by

$$
\hat{\mathbf{M}}(\mathbf{x}, t)=h^{3} \sum_{\mathbf{k}=\mathbf{1}}^{\mathbf{N}} \mathbf{M}_{\mathbf{k}}(t) \delta\left(\mathbf{x}-\mathbf{x}_{\mathbf{k}}(t)\right)
$$

where $h$ is the grid size, $\mathbf{x}_{\mathbf{k}}(\mathbf{t})$ gives the position of the magentisation particles, $\mathbf{M}_{\mathbf{k}}(\mathbf{t})$ is the magnetisation of the $k$ th particle and $\delta$ is a smooth, radially symmetric density function with unit mass and compact support. The equations of motion for these particles are

$$
\frac{d\left(\mathbf{M}_{\mathbf{k}}\right)_{i}}{d t}=-\left(\mathbf{M}_{\mathbf{k}}\right)_{j} \partial_{i} u_{j}, \quad \frac{d \mathbf{x}_{k}}{d t}=\mathbf{u}\left(\mathbf{x}_{\mathbf{k}}\right)
$$

where the subscript $k$ denotes the particle and $i, j$ indicate the component of the vector, while $\mathbf{u}$ is the velocity induced by the magnetisation $\mathbf{M}$. These equations have a Hamiltonian formulation in terms of the Hamiltonian function,

$$
H=\frac{1}{2} \sum_{k=1}^{N} \sum_{j=1}^{N}\left\{\mathbf{m}_{\mathbf{k}} \cdot \mathbf{m}_{\mathbf{j}} \delta\left(\left|\mathbf{x}_{\mathbf{k}}-\mathbf{x}_{\mathbf{j}}\right|\right)+\left(\mathbf{m}_{\mathbf{j}} \cdot \nabla\right)\left(\mathbf{m}_{\mathbf{k}} \cdot \nabla\right) \Psi\left(\left|\mathbf{x}_{\mathbf{k}}-\mathbf{x}_{\mathbf{j}}\right|\right)\right\}
$$

where $\mathbf{m}_{\mathbf{k}}=\mathbf{h}^{\mathbf{3}} \mathbf{M}_{\mathbf{k}}$ and $\Psi$ is the solution of $\triangle \Psi=\delta$; and Hamilton's equations,

$$
\frac{d \mathbf{p}_{k}}{d t}=\frac{-\partial H}{\partial \mathbf{q}_{k}}, \frac{d \mathbf{q}_{k}}{d t}=\frac{\partial H}{\partial \mathbf{p}_{k}}
$$

where $\mathbf{p}_{k}=\mathbf{m}_{k}$, and $\mathbf{q}_{k}=\mathbf{x}_{k}$. Oseledet [10] and Luchini have developed similar Hamiltonian formulations for $3 \mathrm{D}$ ideal fluids.

There is some important differences between first order N-body problems such as $2 \mathrm{D}$ vortex dynamics, and second order problems such as the Newtonian N-body problems, and the Hamiltonian system (7). The second order systems have a phase-space whose dimension is twice that of the configuration space; in first order systems, the dimensions of the phase and configuration spaces are equal. This makes 2 nd order problems harder. The system (7) has all the complexities of the Nbody problems in celestial mechanics. I will discuss some of the techniques used in the gravitational problems to this problem.

\subsection{Singular limit or point magnetisation particles}

First, consider the singular limit where the density function is the dirac delta function. The resulting Hamiltonian for the point particle approximation,

$$
H=\frac{1}{2} \sum_{j=1}^{N}\left|\mathbf{p}_{\mathbf{j}}\right|^{\mathbf{2}}-\sum_{\mathbf{j}<\mathbf{k}}^{\mathbf{N}}\left\{\frac{\mathbf{3} \mathbf{p}_{\mathbf{j}} \cdot\left(\mathbf{q}_{\mathbf{j}}-\mathbf{q}_{\mathbf{k}}\right) \mathbf{p}_{\mathbf{k}} \cdot\left(\mathbf{q}_{\mathbf{j}}-\mathbf{q}_{\mathbf{k}}\right)}{\left|\mathbf{q}_{\mathbf{j}}-\mathbf{q}_{\mathbf{k}}\right|^{\mathbf{5}}}-\frac{\mathbf{p}_{\mathbf{j}} \cdot \mathbf{p}_{\mathbf{k}}}{\left|\mathbf{q}_{\mathbf{j}}-\mathbf{q}_{\mathbf{k}}\right|^{\mathbf{3}}}\right\}
$$

has a simpler form than the original Hamiltonian (7) for a general density function. Note that the three degrees of freedom problem here is not integrable in general. In order to proceed, I will derive an analogue N-body problem for this Hamiltonian: first, note that the momenta $\mathbf{p}_{j}$ correspond to small vortex loops with circulation $\Gamma_{j}>0$ and area element $\mathbf{A}_{j}$. For the same loop, opposite circulations are represented by vectorial area elements which are negative of one another. Treating 
the $\Gamma_{j}$ like masses, and the $\mathbf{A}_{j}$ like velocities, the analogue N-body probelm is given by the following Hamiltonian:

$$
\begin{aligned}
H & =\frac{1}{2} \sum_{j=1}^{N} \Gamma_{j}\left|A_{j}\right|^{2}-\sum_{\mathbf{j}<\mathbf{k}}^{\mathbf{N}}\left\{\frac{3 \Gamma_{j} \Gamma_{k}\left[A_{j} \cdot\left(r_{j}-r_{k}\right)\right]\left[A_{k} \cdot\left(r_{j}-r_{k}\right)\right.}{\left|r_{j}-r_{k}\right|^{5}}-\frac{\Gamma_{j} \Gamma_{k} A_{j} \cdot A_{k}}{\left|r_{j}-r_{k}\right|^{3}}\right\} \\
& =\frac{1}{2} \sum_{j=1}^{N} \Gamma_{j}\left|\mathbf{v}_{j}\right|^{2}-\sum_{j<k}^{N}\left\{\frac{3 \Gamma_{j} \Gamma_{k}\left[\mathbf{v}_{j} \cdot\left(\mathbf{r}_{j}-\mathbf{r}_{k}\right)\right]\left[\mathbf{v}_{k} \cdot\left(\mathbf{r}_{j}-\mathbf{r}_{k}\right)\right.}{\left|\mathbf{r}_{j}-\mathbf{r}_{k}\right|^{5}}-\frac{\Gamma_{j} \Gamma_{k} \mathbf{v}_{j} \cdot \mathbf{v}_{k}}{\left|\mathbf{r}_{j}-\mathbf{r}_{k}\right|^{3}}\right\}
\end{aligned}
$$

where I have used $\mathbf{v}_{j}$ to denote the velocities and $\mathbf{r}_{j}$ to denote the positions of the particles. Clearly, the second sum in the Hamiltonian represents a velocity-dependent potential energy. The range of the interaction through this potential can be obtained by rewriting it in the form:

$$
U=\sum_{j<k}^{N}\left\{\frac{3 \Gamma_{j} \Gamma_{k} v_{j} v_{k} \cos \theta_{j} \cos \theta_{k}}{r_{j k}^{3}}-\frac{\Gamma_{j} \Gamma_{k} v_{j} v_{k} \cos \theta_{j k}}{r_{j k}^{3}}\right\}
$$

where the common denominator $r_{j k}^{3}$ gives the decay rate of the interaction. Since the effective force $f_{j}=\nabla_{j} U$, the force of interaction is inversely proportional to the fourth power of the separation $r_{j k}$ of the particles. This answers a question of Alexandre Chorin at the conference. I am not able at this point to fully answer his second question on the convergence of the Mayer cluster expansion, given that the decay rate is fourth order. Further discussion of related convergence issues can be found in the sections on statistics. The variables $\theta_{j}, \theta_{j k}$ denote respectively the angle between $v_{j}$ and $r_{j}-r_{k}$, and the angle between the velocities $v_{j}$ and $v_{k}$.

An interesting question for this N-body problem of point particles is the type of collisions that may occur. Conjecture: the set of initial data which result in collisions has measure zero in $6 \mathrm{~N}$-dimensional phase space (cf. Saari [12]).

\subsection{Symmetries and first integrals}

From the translational symmetry, one gets the conservation of "linear momentum",

$$
\sum_{j=1}^{N} \mathbf{p}_{j}=\sum_{j=1}^{N} \mathbf{m}_{j}
$$

where $\mathbf{m}_{j}$ denotes the magnetisation of the $\mathrm{j}$ th particle. The conservation of the Hamiltonian or the energy of the problem corresponds to time-translation symmetry. By closely inspecting the structure of the potential energy, $U$, one notes that it is invariant under the $O(3)$ group of symmetries. Thus, the conservation of "angular momentum",

$$
\sum_{j=1}^{N} \mathbf{q}_{j} \times \mathbf{p}_{j}=\sum_{j=1}^{N} \mathbf{x}_{j} \times \mathbf{m}_{j}=\sum_{j=1}^{N} \Gamma_{j}\left(\mathbf{r}_{j} \times \mathbf{A}_{j}\right)
$$

which corresponds to the fluid-mechanical "impulse". Like the Newtonian N-body, this magnetisation N-body problem has seven conserved quantities.

\subsection{Two-body problem and two small vortex rings.}

The analogue Keplerian problem for $N=2$ is completely integrable because the number of conserved quantities is sufficient. Like the Newtonian case, the general three body problem is not 
integrable. The Hamiltonian in this case is:

$$
H=\frac{1}{2}\left(v_{1}^{2}+v_{2}^{2}\right)-\frac{3 v_{1} v_{2} \cos \theta_{1} \cos \theta_{2}}{r_{12}^{3}}+\frac{v_{1} v_{2} \cos \theta_{12}}{r_{12}^{3}}
$$

where the speeds are denoted by $v_{1}, v_{2}$. Let's examine the simple case where the velocities of the two particles are in the same line as that joining them; in this case, $\cos \theta_{1,2}= \pm 1=\cos \theta_{12}$. The motion remains on a line, and the dynamics of the analogue Kepler problem translates into the motion of two parallel vortex rings which are perpendicular to the line joining their centers. The two rings must remain in this configuration and their diameters are determined by the speeds of the analogue particles.

Another interesting motion for the analogue 2-body problem is the circular two particle solution where the two equally massive particles move in circles about their common center of mass. This corresponds to two vortex rings which have opposite circulations but the same diameters, and which lie in the same plane, separated by a distance greater than the diameter. This common plane now rotates at constant angular velocity about the perpendicular bisector in the plane of the line joining the centers of the two rings. In both the in-line as well as the circular motions, the vortex rings do not deform but remain perfectly circular. In this approximation, only the orientation and the diameters of the rings may change since each ring is now represented by only three degrees of freedom.

Besides the above examples of completely integrable motions, there are others for the two-body problem, where the two particles moves in elliptical orbits. Quasi-periodic motions involving a central massive particle, and $n$ less massive planets, can be set up by perturbing the completely integrable system of $n$ decoupled two-body problems that consist of a planet and the sun. The small parameters here are the inclinations of the orbits of the planets and their eccentricities, and the ratio of "planetary masses" to central "mass". When the n-planetary motions are coupled again, the KAM-theorem predicts that the behaviour of the "solar system" is stable and quasi-periodic.

To set up quasi-periodic motions when the number of particles involved is very large, one must use a powerful set of canonical transformations called the "generalized Jacobi variables", which are generated graph-theoretically. This procedure is called the "combinatorial perturbation method", and will be discussed in a later section in the context of the Kirchhoff Hamiltonian. The Jacobi variables come in two related forms, one for $2 \mathrm{D}$ problems and the other, for $3 \mathrm{D}$ problems like the magnetic N-body problem.

\section{Statistical questions}

\subsection{Infinite sums and the dynamics of infinite particle systems}

One important question which needs to be posed in the infinite particles system for the point particle Hamiltonian is: Does the right hand side of $\frac{\partial}{\partial t} \mathbf{p}_{j}=\frac{\partial}{\partial \mathbf{r}_{j}} U$ or the force $f\left(\mathbf{r}_{j}\right)$ on the $\mathbf{j}$-th particle converge? Since the interaction force decays in inverse proportion to the fourth power of the distance between particles (9), one must evaluate the force on the $\mathrm{j}$-th particle by taking infinite sums over all other particles. These sums may converge if the number of particles, $N(j, r, t)$ in a thin spherical shell of unit thickness at distance $r$ from the $j$ th particle, does not exceed $h(r)^{\sim} O\left(r^{3-\varepsilon}\right)$. This holds if the density of particles per unit volume is uniformly bounded, for in that case, $h(r)=O\left(r^{2}\right)$ by a simple calculation of the volume of a thin spherical shell of radius $r$. The real issue however, is, what initial distributions of points in phase space will evolve under the point particle Hamiltonian, and keep $N(r, t)<h(r)$ for all time $t>0$. In the state of statistical equilibrium, what is the measure of those initial points which violate the above density bounds, 
after some time $t$ ? If it can be shown that such "bad" sets have zero Gibbs measure, then the time evolution of the infinite point particle system has physical or statistical meaning. One can then study the existence, uniqueness, and regularity of such motions. In the case of the magnetic point particle problem, the difficulty is compounded by the fact that the interaction force is velocity dependent. This means that uniform bounds on velocities are needed to control the growth of the above infinite sums. Velocity bounds are also needed to control the particle densities at $t>0$ given the initial values of the densities. In the usual kinetic theory, such velocity bounds are ridiculous because the velocities must satisfy a Maxwellian distribution. In the current context, the velocities of the analogue N-body problem translates into areas of the vortex loops, and bounds on these quantities are perhaps less disturbing. After all, the derivation of the magnetic Hamiltonian model is based on small vortex loops. There is a good possibility that the equilibrium distribution of velocities for the Hamiltonian (8) is not Maxwellian due to the long range of the interaction force, and the fact that the interaction potential depends on velocities. Some results for one-dimensional infinite systems are due to Lanford [5], and Ruelle [11]. It would be very interesting to have this problem worked out for the vortex loop gas.

Similar summations are discussed by Greengard and Rokhlin [2] in the context of fast numerical methods for evaluating the electrostatic force at a given point. Such problems also arise in homogenisation techniques for studying the bulk properties of multiphase materials [4].

\subsection{Non-interacting particles}

When the interaction between point magnetic particles is turned off, the resulting Hamiltonian is

$$
H=\frac{1}{2} \sum\left|\mathbf{p}_{j}\right|^{2}=\frac{1}{2} \sum v_{j}^{2}=1 / 2 \sum A_{j}^{2}
$$

where the masses (circulations) $\Gamma_{j}$ has been set to unity. Following the ideal gas case in kinetic theory, the Hamiltonian defines spherical energy surfaces in $3 \mathrm{~N}$-dimensional space. For $H=E$, the radius of the sphere is $\sqrt{2 E}$, and its area is $\Omega=(2 K E)^{(3 N-1) / 2}$, where $\mathrm{K}$ is a constant which depends on the dimension of the space. The partition function

$$
Z=\int \ldots \int d \mathbf{r}_{1} \ldots d \mathbf{r}_{N} \int \ldots \int d \mathbf{p}_{1} \ldots d \mathbf{p}_{N} \exp \left(\frac{-1}{2} \sum_{j=1}^{N} p_{j}^{2}\right)
$$

now gives all the important thermodynamic quantities:

$$
\begin{aligned}
\Psi & =-T \log Z \\
S & =-\frac{\partial \Psi}{\partial T}
\end{aligned}
$$

where $\Psi$ is the free energy, $S$ is the entropy, and the temperature is given by:

$$
T=\left(\frac{d S}{d E}\right)^{-1}=\frac{2 E}{3 N}
$$

\subsection{Mayer cluster expansions}

Another important statistical issue is the derivation of equations of state for the vortex-loop gas when the interaction is switched on. The direct Mayer expansions will not do here because of the velocity dependent potentials in (8). Moreover, can the inverse quartic force law support any 
form of diagrammatic integration techniques [14]? The classical Mayer expansion for the partition function can be summarised as follows:

$$
\begin{aligned}
Z(V, T, N) & =\int \cdots \int_{V \ldots \ldots . V} d \mathbf{r}_{1} \ldots d \mathbf{r}_{N} \cdot \int \cdots \int d \mathbf{p}_{1} \ldots d \mathbf{p}_{N} \exp [-\beta H] \\
& =\left(1 / N ! \lambda^{3 N}\right) \int \cdots \int_{V \ldots \ldots . V} d \mathbf{r}_{1} \ldots d \mathbf{r}_{N} \prod_{i<j}^{N}\left(1+f_{i j}\right) \\
& =\left(1 / N ! \lambda^{3 N}\right) \int \cdots \int_{V \ldots \ldots . . V} d \mathbf{r}_{1} \ldots d \mathbf{r}_{N}\left(1+f_{12}+f_{13}+f_{23}+f_{12} f_{23}+\cdots ;\right.
\end{aligned}
$$

the integration in momentum $3 \mathrm{~N}$ space produced the factor $\lambda^{3 N}$, and

$$
f_{i j}=f\left(\left|\mathbf{r}_{i}-\mathbf{r}_{j}\right|\right)=\exp \left[\frac{1}{k T} \phi\left(\left|\mathbf{r}_{i}-\mathbf{r}_{j}\right|\right)\right]-1
$$

where $\phi$ is the interaction potential between the $\mathrm{i}$-th and $\mathrm{j}$-th particles. In the magnetic Hamiltonian, the total interaction potential is given by $U\left(v_{1}, \ldots, v_{N} ; r_{1}, \ldots, r_{N}\right)$, Eq. 9. The quadratic dependence on the speeds $v_{j}$ can to some extent be controlled by the apriori assumption that one is only interested in small values of the speeds because they correspond to the area of the small vortex loops used in the model. The velocity dependence of the interaction potential and its long range bode well for the real issue here: will initially bounded velocity distributions remain bounded under the evolution of the Hamiltonian system (8). I think that this question has a positive answer provided the particle density is simultaneously controlled. The next question is: Does the bounded velocity distributions have an overwhelmingly large measure in equilibrium. This matter has already been raised earlier in the discussion of the existence of the force for an infinite system of interacting particles. In any case, it is clear that much needs to be done to put the statistical vortex problem on a good foundation.

\section{Combinatorial perturbation method}

CPM is based on a class of symplectic transformations (for n-body problems) which are obtained from full binary trees $T(N)$ with $N$ leave. This class of transformations known as Jacobi coordinates, are actually an optimal change to relative positions, accompanied by a change to "relative" momenta. They are well suited for perturbative work on the dynamics of large clusters. As will be discussed later, there is an important non-perturbative aspect of these transformations which play a role in both numerical and analytical studies of vortex dynamics. I will base this sketch of the CPM on the Kirchoff Hamiltonian for 2D vortex dynamics,

$$
H=\sum_{j \neq k} \lambda_{j} \lambda_{k} \log \left|z_{j}-z_{k}\right|
$$

which is a function defined on the complete graph $K(N)$. The new Hamiltonian in terms of the Jacobi variables $\rho_{j}, j=1, \ldots, N-1$,

$$
H^{\prime}=\sum_{j \neq k} \lambda_{j} \lambda_{k} \log \left|C_{j k}\left(\rho_{1}, \ldots, \rho_{n-1}\right)\right|
$$

is a function on the binary tree $T(N)$ used to generate $\rho_{j}$; each term correspond to a leaf $j$ to leaf $k$ path through the internal nodes $\rho_{j}$ which appears in the argument of $\mathbf{C}_{j k}$. This Hamiltonian can be factored using the partial order of $T(N)$; it is possible to write it in perturbation form [7],

$$
H^{\prime}=H_{0}+H_{1}
$$


where $H_{1}$ is a small term. The term $H_{0}$ is an integrable Hamiltonian expressed as $N-1$ decoupled oscillators. The KAM-nondegeneracy and vectorial Melnikov conditions are now combinatorial expressions in terms of the structure of $T(N)$. After some work, quasi-periodic motions for large numbers of $2 \mathrm{D}$ point vortices and vortex blobs, is established. In the physical plane, these quasiperiodic motions correspond to clustered configurations.

\subsection{Diagrammatic expansion}

The above perturbation series (11) can also be represented graph-theoretically in terms of subgraphs of $T(N)$. The first term $H_{0}$ correspond to the subgraph consisting of N-1 isolated internal nodes with no edges, each term in the sum is represented by one of these internal nodes. The first terms in the expansion of $H_{1}$ are each represented by the subtrees rooted at the corresponding internal nodes: (a) the first order terms correspond to isolated internal nodes in each of these subtrees, and (b) the second order terms correspond to pairs of internal nodes in these subtrees. Starting with the Hamiltonian function (10), one writes

$$
H=\sum_{j \neq k} \lambda_{j} \lambda_{k} \log \rho_{s}+\sum_{j \neq k} \lambda_{j} \lambda_{k} \log \left(1+\sum_{l<s} \frac{C_{l}(j, k) \rho_{l}}{C_{s}(j, k) \rho_{s}}\right)
$$

where $s=s(j, k)$ is the highest node in the leaf $j$ to leaf $k$ path, and $l<s$ represent the nodes that are lower than $s$ in that path. Gathering all the $(j, k)$ terms in the first sum that have the same highest node $s$ in the tree $T(N)$, one obtains the decoupled term,

$$
H_{o}=\sum_{s=1}^{N-1}\left(\sum_{s(j, k)=s} \lambda_{j} \lambda_{k}\right) \log \rho_{s}
$$

which correspond to the subgraph of $T(N)$ described above; each isolated internal (non-leaf) node represents one term in the sum over $(N-1)$ terms. Since the tree has a partial order relation which may represent a number of different small parameter scalings (chosen by the reader), one can moreover, expand the logarithms in the second sum, and regroup the terms according to powers of the small parameter $\epsilon$, and the common highest node $s=1, \ldots, N-1$. I will illustrate this procedure using a simple example consisting of only five internal nodes. One can think of this example as being a small subtree in a larger binary tree. The expansion of $(j, k)$ terms whose highest node is the common node $s$,

$$
\sum_{\substack{j=1,2,3 \\ k=4,5,6}} \lambda_{j} \lambda_{k} \log \left(1+\sum_{l=1}^{4} \frac{C_{l} \rho_{l}}{C_{s} \rho_{s}}\right)=\sum_{\substack{j=1,2,3 \\ k=4,5,6}} \lambda_{j} \lambda_{k}\left\{\left[\sum_{l=1}^{4} \frac{C_{l} \rho_{l}}{C_{s} \rho_{s}}\right]+[. .]^{2}+[. .]^{3}+\cdots\right\}
$$

gives symbolically the following terms:

- $\mathrm{O}(\epsilon): 3 / \mathrm{s}+4 / \mathrm{s} \rightarrow$ terms involving $\lambda_{j} \lambda_{k}\left(\frac{C_{3} \rho_{3}}{C_{s} \rho_{s}}+\frac{C_{4} \rho_{4}}{C_{s} \rho_{s}}\right)$, represented graph-theoretically by the labelled subgraph consisting of the disconnected vertices 3 and 4 ,

- $\mathrm{O}\left(\epsilon^{2}\right): 1 / \mathrm{s}+2 / \mathrm{s}+\left[(3 / \mathrm{s})^{2}+(4 / s)^{2}+2(3 / s)(4 / s)\right] \rightarrow$ terms involving $\lambda_{j} \lambda_{k}\left[\frac{C_{1} \rho_{1}}{C_{s} \rho_{s}}+\frac{C_{2} \rho_{2}}{C_{s} \rho_{s}}+\left(\frac{C_{3} \rho_{3}}{C_{s} \rho_{s}}+\right.\right.$ $\left.\frac{C_{4} \rho_{4}}{C_{s} \rho_{s}}\right)^{2}$, represented graph-theoretically by the labelled subgraph consisting of vertices 1,2 , 3 , and 4 , and the 2-loop $(3,4)$. 
This diagrammatic expansion can be based on a number of small parameters such as the ratio between length scales at two adjacent levels in the tree $T(N)$, or the ratio between the circulations of the vortices at two adjacent levels in $T(N)$. The KAM-theory and Nekhoroshev theory together justify the above diagrammatic expansion. To be fair, it must be noted that the small parameters has to be very small in order for the KAM-theory to work. However, the CPM as well as the above diagrammatic expansions can be done without invoking the KAM-theory. In other words, the truncated Hamiltonian series:

$$
H=H_{0}+H_{1}+H_{2}+\cdots+H_{n}
$$

can be the basis of numerical as well as perturbative work on the dynamics of vortex systems. The reader may want to compare this numerical approximation, based on a truncated Hamiltonian, to that proposed by Greengard and Rokhlin [2], where the long-range interaction between particles is approximated by resumming techniques.

\subsection{Self-similarity of binary trees, and clusters}

$T(N)$ are the simplest type of Cayley trees or Bethe lattice. There is clearly a self-similar structure in all infinite full binary trees, where all leaf nodes are at infinity. Also, some full binary trees which are infinite only along certain directions are self-similar in these directions, e.g., the caterpillars. This self-similarity is relevant for the application of Hamiltonian vortex dynamics to turbulence modelling. One can use the self-similarity of the trees and the clusters they represent to model the very fine scales in turbulent flows. The persistence of regular quasi-periodic dynamics in vortex systems implies that large clusters are stable over very long time scales. This phenomena implies that the partial ordering of length scales in these stable clusters are maintained for a long time.

\section{Combinatorics of the Jacobi symplectic transformations}

In this section, I will discuss some aspects of the above canonical transformations, which are nonperturbative in nature. I mean that they are exact symplectic transformations which can be used to rewrite a given $\mathrm{N}$-vortex system without approximations, in a more convenient form for numerical work. Much of this revolve around the explicit algorithms for producing these Jacobi variables, and their combinatorics. Although the following formulae are for the $2 \mathrm{D}$ case, the corresponding expressions for the 3D case can be found in Lim [6].

To every $T(N)$, there belongs $2 N \times 2 N$ real symplectic matrices of the form

$$
\mathbf{S}=\left[\begin{array}{cc}
\mathbf{A} & \mathbf{0} \\
\mathbf{0} & \mathbf{B}
\end{array}\right]
$$

where $\mathbf{A}^{t} \mathbf{D}=\mathbf{I}$ and the $N \times N$ matrices $\mathbf{A}(\mathbf{m}, T(N)), \mathbf{B}(\mathbf{m}, T(N))$ are given by the following entries depending on a vorticity vector $\mathbf{m}=\left(m_{1}, m_{2}, \ldots, m_{n}\right)$ :

$$
\left.\begin{array}{c}
\text { - } A_{j k}=\left|\frac{M_{j}^{+} M_{k}^{-}}{M_{j}}\right|^{1 / 2}\left\{\begin{array}{ll}
\operatorname{sgn}\left(m_{k}\right) \frac{\sqrt{\left|m_{k}\right|}}{M_{j}^{+}} & \text {if } k \in \Lambda^{+}(j), \\
-\operatorname{sgn}\left(m_{k}\right) \frac{\sqrt{\left|m_{k}\right|}}{M_{j}^{-}} & \text {if } k \in \Lambda^{-}(j), \\
0 & \text { if } k \notin \Lambda(j),
\end{array} \quad j=1, \ldots, N-1,\right.
\end{array}\right\} \quad k=1, \ldots, N,
$$




$$
\begin{gathered}
D_{j k}=\operatorname{sgn}\left(\frac{M_{j}^{+} M_{j}^{-}}{M_{j}}\right)\left|\frac{M_{j}^{+} M_{j}^{-}}{M_{j}}\right|^{1 / 2}\left\{\begin{array}{ll}
\frac{\sqrt{\left|m_{k}\right|}}{M_{j}^{+}} & \text {if } k \in \Lambda^{+}(j), \\
\frac{-\sqrt{\left|m_{k}\right|}}{M_{j}^{-}} & \text {if } k \in \Lambda^{-}(j), \\
0 & \text { if } k \notin \Lambda(j),
\end{array} \quad j=1, \ldots, N-1,\right. \\
D_{n k}=\frac{\sqrt{\left|m_{k}\right| \operatorname{sgn}\left(M_{n-1}\right)}}{\left|M_{n-1}\right|^{1 / 2}}, \quad k=1, . ., N,
\end{gathered}
$$

where

$$
M_{j}^{ \pm}=\sum_{k \in \Lambda^{ \pm}(j)} m_{k,} \quad M_{j}=\sum_{k \in \Lambda(j)} m_{k}
$$

and $\Lambda^{ \pm}(\mathbf{s})=\{$ leaf $\mathbf{j}$ below node $\mathbf{s}$ in $T(N)$, and connected to it via the right(left) branch $\}$,

$$
\begin{array}{rr}
\Lambda(\mathbf{s})=\Lambda^{+}(\mathbf{s}) \cup \Lambda^{-}(\mathbf{s}), & \mathbf{s}=\mathbf{1}, \ldots, \mathbf{N}-\mathbf{1}, \text { provided } \\
& \mathbf{M}_{j}^{ \pm} \neq \mathbf{0}, \mathbf{j}=\mathbf{1}, \ldots, \mathbf{N}-\mathbf{1} \text { and } \mathbf{M}_{n-1} \neq \mathbf{0} .
\end{array}
$$

The sign-pattern for the binary tree $\mathrm{T}(5)$ depicted in figure 1 is given below:

$$
H(T(5))=\left[\begin{array}{ccccc}
-1 & 1 & 0 & 0 & 0 \\
0 & 0 & 0 & -1 & 1 \\
0 & 0 & -1 & 1 & 1 \\
-1 & -1 & 1 & 1 & 1 \\
1 & 1 & 1 & 1 & 1
\end{array}\right]
$$

The above algorithm for S-pairs (A, D) has the following consequences:

1. vorticity vectors of one sign automatically satisfy the above condition, and

2. some vorticity vectors of mixed signs are incompatible with a given tree.

The underlying combinatorial structure of the symplectic matrix $\mathrm{S}$ is the common sign-pattern of the matrices $\mathbf{A}$, and $\mathbf{D}$ in the case of vorticity vectors of one sign. This sign-pattern of $0, \pm 1$, denoted by $\mathbf{H}[T(N)]$ is a complete description of the tree, $T(N)$, but unlike the usual incidence or adjacency matrix representation of graphs:

1. the sign pattern is a totally unimodular matrix, an example of a matroid, and

2. it is sign-nonsingular, to be defined next (cf. Lim [8]).

\subsection{Sign-nonsingular patterns and noneven digraphs}

A square $N \times N$ matrix $H$ of $0, \pm 1$ entries is sign-nonsingular if every real matrix with its signpattern equal to $H$ has nonzero determinant. A digraph $G$ is noneven if there is a weighting of its arcs by 0 and 1 such that every cycle has noneven weight. The weight of a cycle is the sum of the weights of its arcs. These two definitions are equivalent via the weighted adjacency matrix $H$ of the weighted digraph $G^{\prime}$.

Theorem 1. The matrices $\mathbf{H}[T(N)]$ for each full binary tree are sign-nonsingular (cf. Lim [8]). 


\subsection{List of questions}

For each tree, how many different symplectic pairs $(\mathbf{A}, \mathbf{D})$ are there? Are there other algorithms for getting $S$-pairs from $\mathbf{H}[T(N)]$ besides one above? What if any sign-nonsingular patterns always yield $S$-pairs, or require $S$-pairs? These have the advantage of generating $\mathbf{N}^{2}$-parameter family of symplectic matrices.

The answer to the first two questions is given by:

Theorem 2. There is precisely an $(N-1)$-parameter family of $S$-pairs for each tree (cf. D. Schmidt Ph.D Thesis RPI 1995).

The generalised Jacobi algorithm given above has $N-1$ independent parameters, and is thus optimal. In other words, there are no other $S$-pairs.

To answer the third question in the above list, I will define the following concept: A signnonsingular matrix $H$ requires $S$-pairs if the inverse-transpose of every matrix $A$ with that signpattern is a matrix $D$ with the same sign-pattern.

Theorem 3. The only noneven digraphs that require $S$-pairs are isolated 2-cycles on $N$ vertices (cf. Schmidt and Lim [13]).

\section{Conclusion}

An interesting connection between the dimer problem in condensed matter physics and a symmetric version of the sign-nonsingular problem above was recently discovered by this author (cf. Lim [9]): a complete characterization of the symmetric noneven digraphs can now be given in terms of Kastelyn's theorem on dimer solutions of the Ising model (cf. Kastelyn [3]). The literature on phase transitions and polymer physics, abounds in enumeration problems for various subgraphs of lattices. For instance, one is interested in the number of self avoiding walks of a certain diameter in the estimation of the Flory exponent in polymer physics. In combinatorial solutions of the 2D Ising model, the number of dimer coverings of the Ising lattice plays an important role. This enumeration problem can be solved if a sign-nonsingular Pfaffian exists for the lattice, that is, if the lattice graph is noneven in a symmetric sense. On the other hand, (fully) noneven digraphs are precisely the graphs which can be weighted so that its adjacency matrix is sign-nonsingular. The corresponding enumeration problem that can be solved for noneven digraphs, is the number of cycle coverings, because to each term in the determinant sum (over the permutations) of the sign-nonsingular adjacency matrix which must have the same sign, there belongs a set of disjoint cycles which cover the graph, whence the number of cycle coverings equals the determinant.

\section{References}

[1] Chorin, A.J., Vorticity and Turbulence, Springer-Verlag, New York, 1994.

[2] Greengard, L. and Rokhlin, V., "A Fast Algorithm for Particle Simulation," J. Comp. Phys., 73, pp. 325, 1987.

[3] Kastelyn, P., "Graphs and crystal physics," Graph Theory and Theoretical Physics, edited by F. Harary, 1967.

[4] Kohn, R., Random Media and Composites, SIAM, 1989. 
[5] Lanford III, O., "Time-Evolution of Infinite Classical Systems," in Math. Aspects of Stat. Mech., Amer. Math. Soc., pp. 65-76, 1972.

[6] Lim, C.C., "Binary Trees, Symplectic Matrices, and the Jacobi Coordinates of Celestial Mechanics," Arch. Rat. Mech Anal., 115, pp. 153-165, 1991.

[7] Lim, C.C., "A Combinatorial Perturbation Method and Whiskered Tori in Vortex Dynamics," Physica, 64D, pp. 163-184, 1993.

[8] Lim, C.C., "Nonsingular Sign-Patterns and the Orthogonal Group," Linear Algebra and its Applic., 184, pp. 1-12, 1993.

[9] Lim, C.C., "Kastelyn's Theorem on Dimers and Symmetric Noneven Digraphs," Proc. 27th SE Conf. on Graph Theory, Combinatorics and Computations, Baton Rouge, February, 1996.

[10] Oseledet, V.I., "On a New Way of Writing the Navier-Stokes Equations: The Hamiltonian Formalism," Comm. Moscow Math. Soc. 1988, transl. in Russ. Math. Surveys, 44, pp. 210$211,1989$.

[11] Ruelle, D., "States of Classical Statistical Mechanics," J. Math. Phys., 8, pp. 1657-1668, 1967.

[12] Saari, D., "Singularities and Collisions of Newtonian Gravitational Systems," Arch. Rat. Mech. \& Analysis, 49 (4), pp. 311-320, 1973.

[13] Schmidt, D. and Lim, C.C., "Full Sign-Invertibility and Symplectic Matrices," Linear Algebra and its Applic., Fall, 1995.

[14] Uhlenbeck, G.E. and Ford, G.W., Lectures in Statistical Mechanics, Amer. Math. Soc., 1963. 


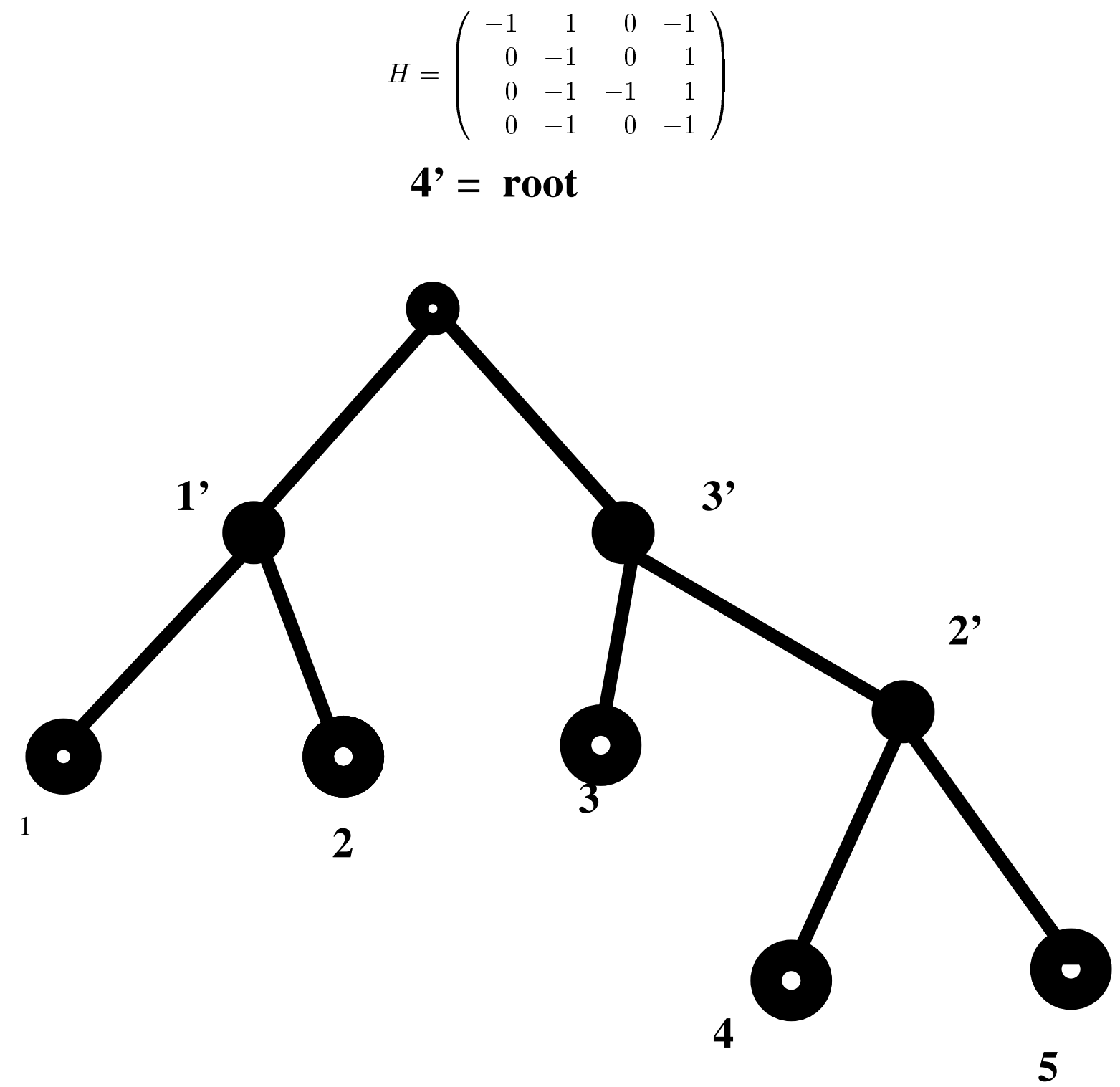

Figure 1: Full binary tree T(5) 